lying behind the regular division of the cells of the ovum, their division into separate groups and the accurate timing of all these changes ? Much patient work has been expended on the elucidation of these questions and advance has been made, but we have not yet come at the truth, and much research remains to be carried out.

\title{
THE RELATION OF OPHTHALMOLOGY TO THE THEORY OF VISION*
}

BY

\author{
F. W. EDRIDGE-GREEN, M.D., F.R.C.S. \\ OPHTHALMIC SURGEON, SOUTH LONDON RECRUITING AREA
}

IF a theory is not only an explanation but actually true, it ceases to be a theory and becomes what is far more important, a fact.

The theory of vision is as follows :-A ray of light impinging on the retina liberates the visual purple from the rods and a photograph is formed. The rods are concerned only with the formation and distribution of the visual purple, not with the conveyance of light impulses to the brain. The ends of the cones are stimulated through the photo-chemical decomposition of the visual purple by light, and a visual impulse is set up which is conveyed through the optic nerve fibres to the brain. The character of the stimulus differs according to the wave-length of the light causing it. In the impulse itself we have the physiological basis of the sensation of light, and in the quality of the impulse the physiological basis of the sensation of colour. But though the impulses vary in character according to the wave-length of the light causing them, the retinocerebral apparatus is not able to discriminate between the character of adjacent stimuli, not being sufficiently developed for the purpose. This power of discrimination varies with individuals, and hence the different varieties of colour perception which are found.

It is not intended to deal with the overwhelming physiological, physical, and psychological facts in support of the theory, as these have been given elsewhere, many of the facts being so inexplicable that no previous explanation had been attempted. Houstoun $\dagger$ in a very able paper, has completely answered any physical objections which might have been made and has shown that the theory could be deduced from physical facts with the aid of mathematics.

It is the purpose of this paper to show that every possible aberration of function, in accordance with the theory, is represented by a disease of the eye, none of which have hitherto had a satisfactory and accepted explanation.

\footnotetext{
*Paper to be read at the Oxford Ophthalmological Congress, July, 1917.

†Proc. Royal Society, A, Vol. XCII, 1916, p. 424.
} 
1. Vision being photo-chemical, there should be defects in accordance with known photo-chemical facts.

2. The rods having the special function of regulating the photochemical sensitiveness of the liquid surrounding the cones, there should be diseases corresponding to aberration of this function.

When the sclerotic, choroid, and pigment cells of the retina are removed, the external fovea is seen as a small basin with four canals leading into it, the whole forming an appearance like a star-fish with curved rays. In normal conditions there is a circulation of lymphatic fluid sensitized by the visual purple from the surrounding parts of the retina into the external fovea and then out through the choroid into the lymphatic spaces of the optic nerve. This retinal circulation is greatly aided by the four canals.

As the retina is thinner in the situation of the canals than at adjacent portions, it would be thought that any obstruction to this retinal lymphatic circulation would tend to dilate these canals and cause the star-fish figure to be obvious on ophthalmoscopic examination. This has been found by Horsley in tumours of the frontal lobe. It would seem a priori that slow obstruction would cause this dilatation, whilst sudden obstruction would cause complete detachment of the retina. Obstruction of this lymphatic circulation may account for many retinal detachments, especially those of the macular region. It occurred to me that the obstruction of this lymphatic circulation by the posterior staphyloma in cases of high myopia would cause the appearance of the star-figure, and, in fact, it is very common in these cases. In one case of high myopia a curious appearance was observable: the star-shaped figure was to be seen in red, with a small black spot in the centre of the fovea. This star appeared red on a white ground, the surrounding choroid having atrophied. Obstruction of this circulation should also tend to cause night blindness, and this we find is common in high myopia.

Nystagmus. - There appear to be two methods by which the sensitized visual fluid is caused to flow into the fovea. The first, when light falls upon the retina external to the fovea, and the other, by movement of the eye. If light fall upon the fovea, and not upon the surrounding portions of the retina, the eye tends to oscillate; this oscillation ceasing when the peripheral portions of the retina are stimulated. This is probably the primary cause of miners' and many other varieties of nystagmus. Nystagmus is also found in conditions in which there is obstruction to central vision, the condition being primarily set up by the desire to obtain better vision by more efficiently sensitizing the fovea by moving the eye.*

*The condition would be greatly accentuated if there were nervous insensitiveness as well. Cridland (The Ophthalmoscope, Vol. II, p. 727) shows that this is the case in miners' nystagmus and that there is contraction of the visual fields. 
Amblyopia.-Any reflex inhibition of the distributing function of the rods, that is, their power of pouring out the visual purple into the surrounding liquid, in accordance with the requirements of the eye, will cause amblyopia, and the removal of the cause, as, for instance, a decayed tooth, may lead to the complete restoration of sight. It is obvious that no ophthalmoscopic changes will be found. It will be noticed that in this case the retina resembles a gland, the function of which may remain in abeyance for a considerable time, and yet in appropriate conditions secrete normally. This may also account for the amblyopia of squint, and on account of the early stage of the life of the individual, the function may remain permanently in abeyance.

Erythropsia.-There is no need for any elaborate theory to explain erythropsia; all photo-chemical substances when acted upon by a bright light have a maximum in their luminosity curves, different from those obtained when acted upon by a feeble light. In the case of a bright light, the maximum is nearer the red end of the spectrum; with a feeble. light it has shifted towards the violet end of the spectrum. In the case, therefore, of visual purple formed under the influence of a very bright light, we should expect it to be more sensitive than normal to the red end of the spectrum, and that erythropsia would be caused, and this is the case.

Night blindness.- It is obvious that if the distributing function of the rods ceases almost entirely when passing from bright to dim illumination we shall have night blindness. We know that the visual purple is restored more rapidly under the stimulation of light than in darkness. There is much variation in individuals in the rapidity with which they can become dark-adapted. It would appear, therefore, that congenital night blindness is due to a defective power of the rods to respond to the stimulus of a feeble light. This may also be brought about by long exposure to a very bright light. Destruction of many of the rods will also have the same efiect. In the case of a poison acting upon the rods we should have blindness at first without ophthalmoscopic appearances, and when vision returned, there should be contraction of the fields of vision and night blindness. A case of quinine poisoning recorded by A. J. Ballantyne* supports this; and he comes to the conclusion that the quinine has a selective action on the rods.

Functional Disturbances.-There are numerous functional disturbances which are easily explained on the theory and many of these facts are now definitely recognized in systems of lighting. Any system of lighting which would produce an unsatisfactory photograph is unsuitable from a visual point of view. Peripheral stimulation of the retina is a necessity and when there is no

${ }^{*}$ British Jl. of Ophthal., March, 1917, p. 153. 
peripheral stimulation, complaints are made of headache and other functional troubles. Those who complain of headaches when only the central portion of the field of vision is illuminated, as, for instance, by a reading lamp, find that they are able to read in comfort when the room is illuminated as well.

Reversal of Colour Fields.-We have an easy explanation of this in the fact that there are two definite physiological processes in the retina, one corresponding to the photo-chemical decomposition of the fluid surrounding the cones which has been sensitized by the visual purple, and the other to the stimulation of the ends of the cones by the decomposition products. Though red light is not nearly so active in bleaching the visual purple as green or blue, when it does produce an effect at all, its action is greater.

Stephenson* makes observations on the transposition of the red and green fields in xerosis conjunctivæ, and states that xerosis is always accompanied by signs of torpor retinæ; therefore we should expect that the action of red would be more affected than that of green. We also find the opposite condition. In hyperæsthesia we should expect to find the red field larger than the blue and this reversal is found in hysteria.

Scintillating Scotoma.-If the supply of sensitized fluid to the fovea ceased we should expect a central scotoma or a fluctuating one if the supply nearly ceased. Higgens found constriction of the retinal arteries in a case examined by him during the attack.

This paper is published in advance so that any member can have an opportunity of bringing forward any fact, if possible, against the theory. Full references to the physiological evidence will be found in Science Progress, January, 1915.

\section{THE APOCRITIC PRINCIPLE AND THE EVOLUTION OF VISUAL PERCEPTIONS $\uparrow$ \\ BY \\ J. Herbert Parsons, LONDON.}

WHEN crystals separate out from a solution, certain molecules detach themselves from all others of a different kind, group themselves in a particular manner, and produce or create a mass of material which, while retaining essential characteristics of the original molecules, becomes endowed with entirely new physical properties.

\footnotetext{
"The Ophthalmoscope, March, 1912.

$\dagger$ Communicated to the 1917 Congress of the Ophthalmological Society of the United Kingdom.
} 